\title{
Interactions between Superplasticizer and Release Agents at the Concrete/Formwork Interface
}

\author{
Samir Bouharoun ${ }^{1}$, Yannick Vanhove ${ }^{1}$, Chafika Djelal ${ }^{1}$, Pascale De Caro ${ }^{2}$, Isabelle Dubois ${ }^{3}$ \\ ${ }^{1}$ Laboratoire Génie Civil et géo-Environnement (LGCgE)—Lille Nord de France, IUT de Béthune, Rue de l’Université, Béthune, \\ France; ${ }^{2}$ Université de Toulouse, INP, LCA (Laboratoire de Chimie Agro-Industrielle), ENSIACET, Allée Emile Monso, Institut \\ National de la Recherche Agronomique, Toulouse, France; ${ }^{3}$ Centre de Recherche Lafarge, Rue du Montmurier, St Quentin Fallavier, \\ France. \\ Email: sbouharoun@yahoo.fr
}

Received February $20^{\text {th }}, 2012$; revised March 24 $4^{\text {th }}$, 2012; accepted April 29 ${ }^{\text {th }}, 2012$

\begin{abstract}
Improving the knowledge of rheological and tribological characteristics of fresh concrete is important to contribute to the progress of construction sites and the final quality of the work. The objective of this study is to identify the effect of a superplasticizer based on polycarboxylic ether on the tribological behavior of fresh concrete at the concrete/formwork and concrete/oil/formwork interfaces. Friction tests on fresh concrete were carried out using a plan/plan tribometer. In order to study the behavior of the superplasticizer close to the formwork, three concretes with $30 \%$ of paste and different dosage of superplasticizer were formulated. The results show that the increase of the dosage of superplasticizer reduces the friction stress. The properties of the superplasticizer generate a deflocculating action of concrete grains and lead to a stabilisation of the soap-oil micellae present in the vicinity of the formwork. Thus, the efficiency of superplasticizer depends on the quantity of fines, on the quantity of soap formed and so, on the release agent formulation.
\end{abstract}

Keywords: Tribology; Concrete/Formwork Interface; Concrete/Oil/Formwork Interface; Superplasticizer; Release Agent; Physicochemical

\section{Introduction}

New concretes must simultaneously meet strength and workability requirements. These characteristics appear irreconcilable: if superplasticizer is not added, the increase in strength is achieved by reducing the quantity of water in the mixture, to the detriment of fluidity, whereas workability can only be improved by increasing the quantity of mixing water, thus reducing the final strength of the material [1]. The use of superplasticizer ensures that the concrete is fluid enough for easy placement in formworks. However, few studies have been done on the interaction between superplasticizer and release agents, whose role is to ensure unblemished casting surfaces and formwork removal without snagging or dusting. But if these oils are used incorrectly, or are incompatible with certain constituents of the concrete, they may cause numerous problems such as staining and bugholes, and even cause pores in the concrete.

At present, mineral oils are most widely used. But these products have low biodegradability and cause user discomfort. To resolve these drawbacks, vegetable formulations have been developed.

These release agents differ chemically from the hy- drocarbons that compose mineral oils. The latter have an essentially physical mode of action. They adhere to the formwork and, as they are hydrophobic, the fresh concrete does not make contact with the walls formwork. Vegetable release agents, however, have a physicochemical action that has not been extensively described. In the presence of superplasticizer in concrete, these products acquire new functional properties and a mode of action specific to them. We therefore conducted a study to explain the mechanisms that occur at the concrete/oil interface when concrete is placed.

The mechanisms were highlighted using a tribological approach. The main purpose of this paper is to study the influence of superplasticizer on friction stress in the presence of a mineral or vegetable release agent. Friction tests were performed using a plane/plane tribometer on one ordinary concrete and on two concretes with different dosages of superplasticizer.

\section{Experimental Methods}

\section{Plane/Plane Tribometer}

The principle of this device was inspired by the box 
shear apparatus used in soil mechanics Figure 1. It can reproduce the conditio Université de Toulouse, INP ns encountered by manufacturers of concrete walls and precast elements. In particular, it can create sliding contacts between concrete, release agent and formwork $[2,3]$.

Two $120 \mathrm{~mm}$ diameter cylinders, with the concrete inside, were placed either side of a metal plate. The sample-holders were fitted with a gasket system to prevent water egress. The plate was set in motion using a motor coupled to an endless screw. Plate travel was $800 \mathrm{~mm}$. The concrete was pressured against the plate by a jack.

The frictional, or tangential, stress was calculated by the equation:

$$
\tau_{\mathrm{f}}=\frac{\text { Measured force }- \text { Parasitic force }}{\text { Section of sample }- \text { holder }}=\frac{\mathrm{F}_{\text {mes }}-\mathrm{F}_{\mathrm{par}}}{\mathrm{Sc}}
$$

$F_{\text {par }}$ is the resultant of the parasitic frictional forces due to the watertight system against the plate. The area in contact between the concrete and the plate is calculated from the diameter of the sample-holder. In our case, this area is $\mathrm{Sc}=113.1 \mathrm{~cm}^{2}$.

The experimental conditions are as follows:

- The metallic plate was cut out of a wall formwork, its roughness is: $R_{a}=1 \mu \mathrm{m}\left(R_{a}\right.$ being the mean distance between the highest and lowest points of the plate roughness profile) and $\mathrm{R}_{\mathrm{t}}=7 \mu \mathrm{m}\left(\mathrm{R}_{\mathrm{t}}\right.$ being the maximum peak-to-valley height of the roughness profile);

- The plate sliding speed: $1.67 \mathrm{~m} / \mathrm{h}$, which corresponds to concrete placing speed;

- Normal pressure: 30, 50, 70 and $90 \mathrm{kPa}$, which corresponds to the lateral pressure exercised by the concrete on formworks of 1.2, 2, 2.8 and $3.6 \mathrm{~m}$ in height.

\section{Characteristics of the Materials}

\subsection{Concrete}

Two concretes (B30A1 and B30A2) were formulated from an ordinary concrete (B30) with different dosages of superplasticizer. To keep the same workability between the three concretes, the water/(cement + filler)

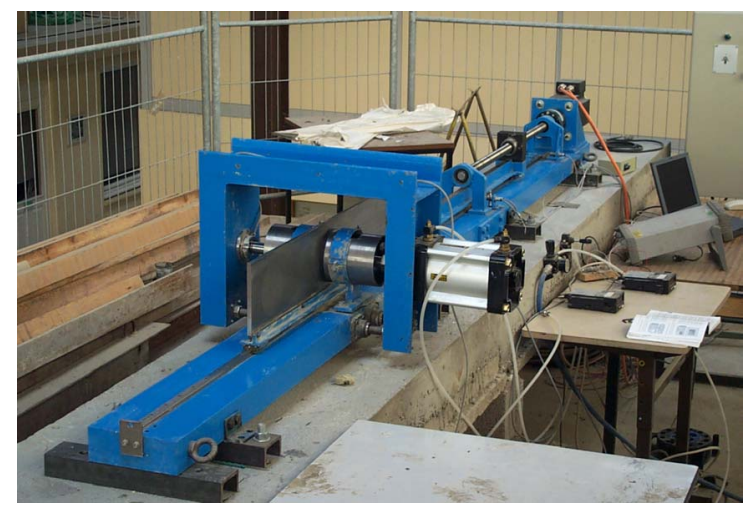

Figure 1. Tribometer. ratio was adapted to obtain a consistency class of S3 (very plastic according to EN 206-1). A slump test was conducted after each mix. The composition of the concretes is given in Table $\mathbf{1}$.

The cementitious material used in all mixtures consisted of a CEM I 52.5 CP2 Type Portland cement with a Blaine fineness of $410 \mathrm{~m}^{2} / \mathrm{kg}$ and a density of $3100 \mathrm{~kg} / \mathrm{m}^{3}$ and a ground granulated blast furnace slag.

Aggregates used to make concrete can reach $12.5 \mathrm{~mm}$ in diameter. Fine particulates are all the sand, cement and filler constituents with a diameter less than $80 \mu \mathrm{m}$. The admixture (GLENIUM 27) used is a high range water reducing admixture of a new chemical generation, based on modified polycarboxylic ether polymers.

In our case, the French standard NF P 18-404 (entitled "Concretes-Design, suitability and inspection testingSpecimen production and conservation) was taken into account. The concrete was mixed from dry materials, using the following procedure Figure 2.

This standard recommends not wetting the mixer, so as to avoid pointless consumption of some of the concrete fines. It is preferable to make a preliminary mix with the

Table 1. Composition and properties of the concretes.

\begin{tabular}{lccc}
\hline \multicolumn{1}{c}{ Concretes } & B30 & B30A1 & B30A2 \\
\hline Paste volume (\%) & 30 & 30 & 30 \\
Cement CEM I 52.5 CP2 $\left(\mathrm{kg} / \mathrm{m}^{3}\right)$ & 248 & 269 & 294 \\
Limestone filler $\left(\mathrm{kg} / \mathrm{m}^{3}\right)$ & 83 & 90 & 98 \\
Sand 0/4 $\left(\mathrm{kg} / \mathrm{m}^{3}\right)$ & 815 & 815 & 815 \\
Crushed aggregate $4 / 8\left(\mathrm{~kg} / \mathrm{m}^{3}\right)$ & 279 & 279 & 279 \\
Crushed aggregate 8/12.5 $\left(\mathrm{kg} / \mathrm{m}^{3}\right)$ & 756 & 756 & 756 \\
HRWRA $(\%$ Sp/C) & - & 0.7 & 1.14 \\
Water $\left(\mathrm{kg} / \mathrm{m}^{3}\right)$ & 189 & 179 & 169 \\
Slump (mm) & 130 & 130 & 130 \\
Water/(cement + Limestone filler) & 0.57 & 0.5 & 0.43 \\
$\quad$ Coarse aggregate/Sand & & 1,27 & \\
\hline
\end{tabular}

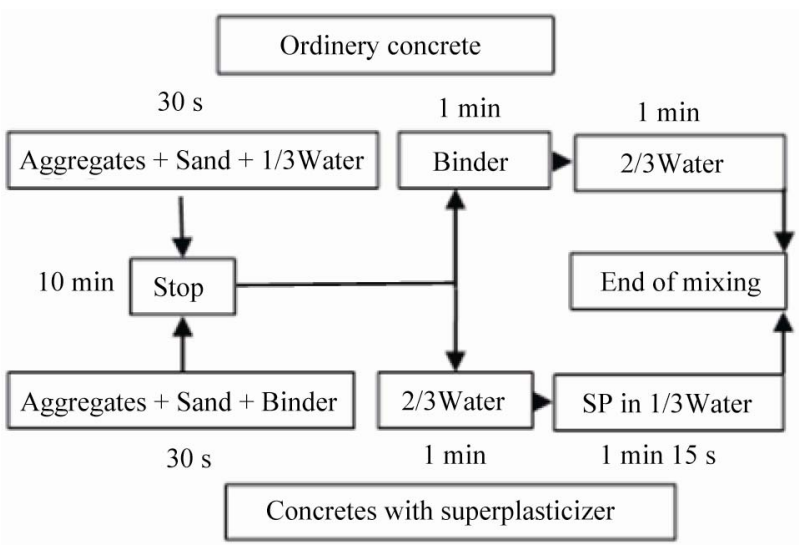

Figure 2. Mixing procedure. 
test concrete, and then discard it.

\subsection{Release Agents}

The two release agents selected for this study belong to the two families of oils on the market: one of petrochemical origin $(\mathrm{Hm})$, and one of vegetable origin $(\mathrm{Hv})$.

Mineral oil (Hm) is a delayed release agent ready-touse, liquid in form and pale yellow in colour. It comprises a primary mineral base with the addition of an acidifier and an anti-corrosion agent (the latter was not considered in the study). This oil, which consists of hydrocarbons, has low biodegradability and can therefore cause water-table pollution.

Vegetable oil (Hv) is a delayed release agent ready-touse. It is also a liquid, and straw-yellow in colour. Besides the oil base, it contains an acidifier and a solvent. All the constituents are vegetable-based for eco-friendliness and user protection. Table 2 lists the properties of these formulations.

The application method of release agents on the wall formwork is particularly important, because it conditions the quality of the concrete facing. The release agent must be applied to a clean surface in an even fashion, to create a continuous, uniform film. On jobsites, the oil is typically sprayed onto the wall formwork, or sprayed and then spread with a rubber squeegee to remove surplus oil and leave a monolayer. For our study, the two release agents were sprayed on using an Eco-spray type sprayer at $20 \mathrm{~cm}$ from the tribometer plate, to prevent an oil surplus and ensure good oil distribution on the plate.

The thickness of the oil films was measured by weighing, according to their method of application. The findings were compared to a more precise measurement conducted using an analysis technique based on alpha radiation [4]. The findings showed good concordance between the two measuring methods.

The oil was sprayed on a circular formwork specimen $3 \mathrm{~cm}$ in diameter.

As the density of the oil and the plate surface area

Table 2. Characteristics of the release agents.

\begin{tabular}{lcc}
\hline \multicolumn{1}{c}{ Release agents } & Vegetable oil & Mineral oil \\
\hline Nature & Liquid & Liquid \\
Reference & $\mathrm{Hv}$ & $\mathrm{Hm}$ \\
Color & yellow & yellow \\
Flash point $\left({ }^{\circ} \mathrm{C}\right)$ & $>100$ & $>100$ \\
Acid number $(\mathrm{mg} \cdot \mathrm{KOH} / \mathrm{g})$ & 5.4 & 1 \\
Hydroxyde number $(\mathrm{mg} \cdot \mathrm{KOH} / \mathrm{g})$ & 1 & 0.85 \\
Density & 0.93 & 0.86 \\
Viscosity at $20^{\circ} \mathrm{C}(\mathrm{mPa} \cdot \mathrm{s})$ & 66.6 & 24.5 \\
Contact angle $\left({ }^{\circ}\right)$ & 18.3 & 12 \\
\hline
\end{tabular}

were known, the thickness of oil could be determined by weighing the specimen.

$$
\mathrm{V}=\mathrm{S} \times \mathrm{e} \Leftrightarrow \mathrm{e}=\frac{\mathrm{V}}{\mathrm{S}} \text { with } \mathrm{V}=\frac{\mathrm{m}}{\rho}
$$

$$
\text { Giving } \mathrm{e}=\frac{\mathrm{m}}{\rho \times \mathrm{S}}
$$

where: $\mathrm{S}$ is the specimen surface area $\left(\mathrm{m}^{2}\right), \rho$ the oil density $\left(\mathrm{kg} / \mathrm{m}^{3}\right)$, $\mathrm{m}$ the weight of the oil film $(\mathrm{kg})$ and e the film thickness (m).

The measurement uncertainty was $\pm 0.15 \mu \mathrm{m}$, taking a measurement error of $5 \%$. For both oils, we obtained a film thickness of about $8 \mu \mathrm{m}$.

\section{Testing and Results}

The tests were first conducted without release agent.

\subsection{Influence of Superplasticizer Dosage at the Concrete/Formwork Interface}

Figure 3 shows the friction stress as a function of contact pressure for each concrete. These tests highlight the influence of superplasticizer dosage.

For our pressure range, friction of the concrete against the metal surface followed Coulomb's law. Fresh concrete, contrary to its appearance, is not a continuous medium. The various constituents of concrete have specific roles during the friction.

The stress applied to the concrete is transferred to the granular phase and the paste formed by the binder (cement, filler). This stress will cause the migration of part of the liquid phase and the fines (of less than $80 \mu \mathrm{m}$ diameter) towards the interface. A lubricating boundary layer, comprising water and fines, then appears at the interface. As there is a small volume of paste (30\%), the flow of the boundary layer depends on the dosage of superplasticizer. This is why the friction stress decreases as the dosage of superplasticizer increases.

The addition of superplasticizer to a concrete scatters

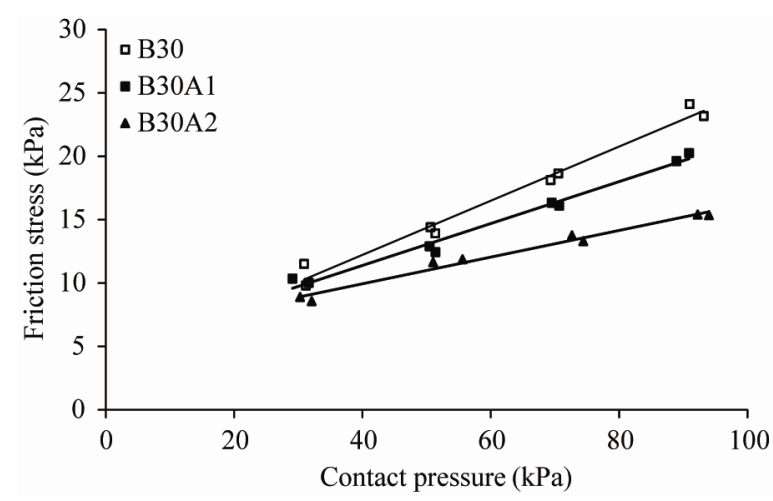

Figure 3. Friction stress as a function of contact pressure. 
the cement grains by the steric effect and electrostatic repulsion [5]. The molecules in the superplasticizer are adsorbed in the surface of the cement particles, imparting negative electrostatic charges. In this way, the cement particles repel one another and are deflocculated Figure 4.

When the superplasticizer dosage increases the dispersing power on the cement grains increases. This property improves concrete flow and, consequently, its creep rate. The presence of superplasticizer reduces inter-grain friction by releasing a greater amount of trapped water.

At the concrete/formwork interface, the boundary layer therefore becomes less rigid. Mobility of the cement and filler grains is facilitated, allowing easier creep of the medium under shear. This reduces the friction stress at the concrete/formwork interface.

\subsection{Influence of the Nature of Release Agents}

Figures 5-7 show the friction stress as a function of contact pressure for the two oils studied. The test done without applying oil was taken as the reference.

Irrespective of concrete composition, the same trends were observed between the reference curve and the two oil curves.

It can be observed that the friction stress decreases in the presence of the oils, and especially of the vegetablebased oil, which confirms the findings in Libessart's dissertation [6]. A new boundary layer comprising water, fines and oil is created. The friction stress trend depends directly on the properties of this fine layer close to the face.

In the presence of the mineral oil, the boundary layer and the aggregates slide on the oil film Figure 8. In this case, the thickness of the oil film is an important parameter $[6,7]$. The thicker the film, the less aggregate comes into contact with the plate.

In the case of the vegetable-based oil, the ester molecules have a strong affinity with the wall formwork. Moreover, the carboxylates formed (soaps) have an amphiphilic structure in the form of a double layer super- posed on the ester layer. This arrangement helps to reduce the friction stress [6,7] Figure 9.

The soaps are calcium carboxylates, more precisely fatty acid salts, which can have two origins:

- A vegetable oil (or an ester) which, in its basic medium, turns into carboxylate by saponification;

- A free fatty acid added to the formulation as an acidifier, which, in basic medium, turns into a fatty acid salt through salification.

In any case, the quantity of soap formed depends on the medium $\mathrm{pH}$ and on the $\mathrm{pKa}$ of the acid, according to the formula:

$$
\mathrm{pH}=\mathrm{pKa}+\frac{\log [\mathrm{B}]}{\log [\mathrm{A}]}
$$

For example, the pKa of oleic acid, a common acidifier, is 4.8. These soaps have a thick texture which settles on the oiled wall formwork.

By their filmogenic power, soaps play a role in reducing friction. The shear at the interface occurs in the soap film, and the boundary layer slides on the soap film Figure 10.

In the case of the mineral-based release agent $(\mathrm{Hm})$, less soap is formed because it comes solely from the acidifier. The soap detaches from the oil film and does not take part in lubrication. The boundary layer then slides on the oil film. The reduction in friction observed at the concrete/Hm/formwork interface is due mainly to the hydrophobic nature of the mineral base, which prevents the concrete from adhering to the formwork. This physical effect is controlled by the thickness of the film.

\subsection{Effect of Concrete Formulation on Release Agent Behaviour}

Figures 11 and 12 show the friction stress as a function of superplasticizer dosage for each of two oils.

Whatever the nature of the release agent, friction decreases when the dosage of superplasticizer increases. Moreover, friction stress is always lower with vegetable-
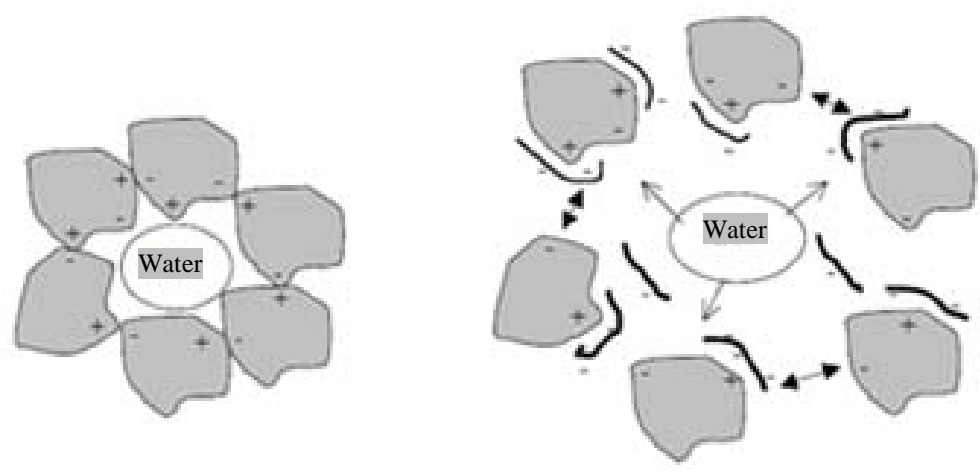

Figure 4. Action of superplasticizers-deflocculation of cement grains [5]. 


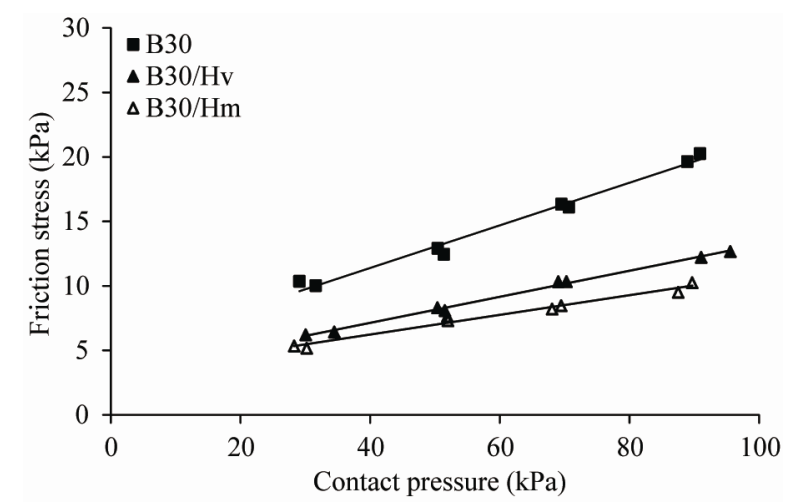

Figure 5. Friction stress as a function of contact pressure for $B 30$ concrete.

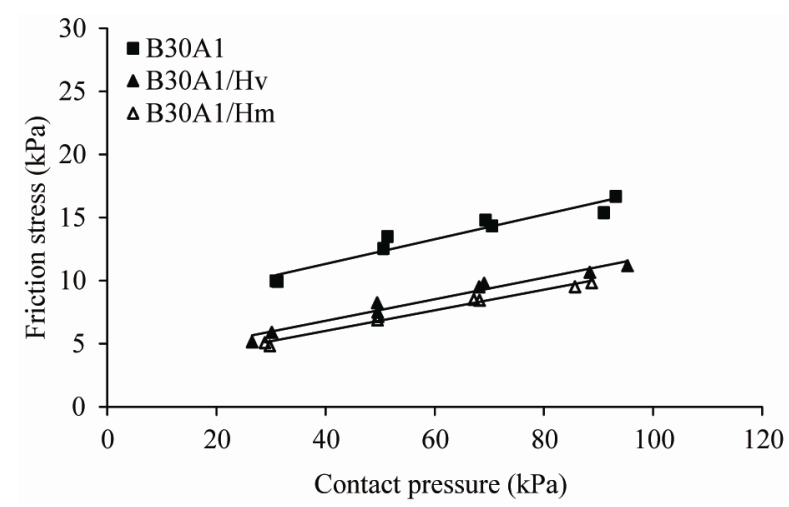

Figure 6. Friction stress as a function of contact pressure for $B 30 A 1$ concrete.

based oils.

The concretes studied have a constant paste volume and a ratio (water/binder) which depends on the dosage of superplasticizer. When this ratio decreases, the quantity of fines increases from $1.12 \%$ to $1.22 \%$ for concretes B30A1 and B30A2 respectively, compared to the ordinary concrete B30.

The extracted interface media show that the surfactant properties of the superplasticizer stabilise the interstitial oil-soap emulsion. This emulsion occupies the boundary layer and increases its viscosity, and thus improves its lubricating properties.

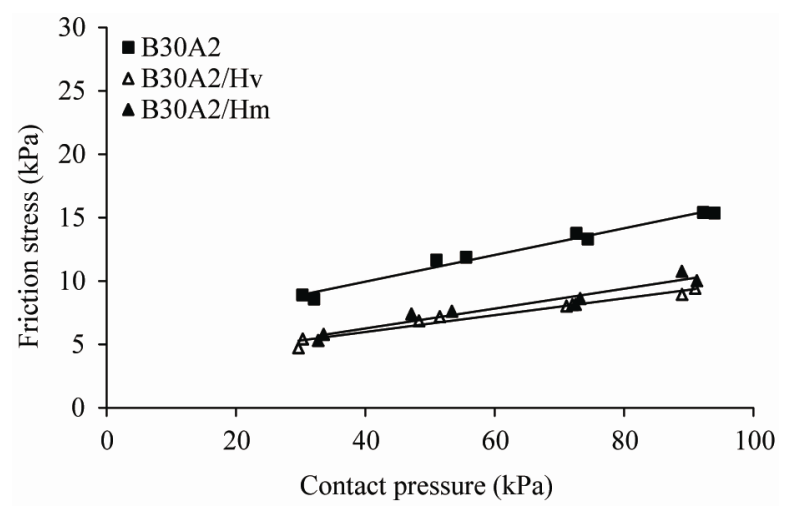

Figure 7. Friction stress as a function of contact pressure for $\mathrm{B} 30 \mathrm{~A} 2$ concrete.

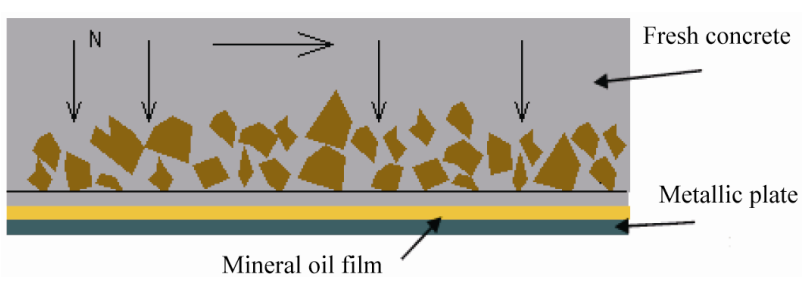

Figure 8. Schematic representation of the mineral-oil film at the concrete/formwork interface.

With vegetable-based oil, the interface is organised in two levels:

- The soap film superposed on the oil film;

- The layer formed by the soap-oil emulsion.

This organisation proves to be particularly effective in reducing friction and hydrophobing the wall formwork.

Although the superplasticizer interacted less with the mineral oil than with the vegetable oil, its role is equally important in the case of the mineral oil. The superplasticizer stabilises the interstitial emulsion, which is less rich in soap, thus having a protective effect on the wall formwork that is characteristic of the mode of action of a mineral oil.

\section{Conclusions}

This study highlighted the role of superplasticizer at the

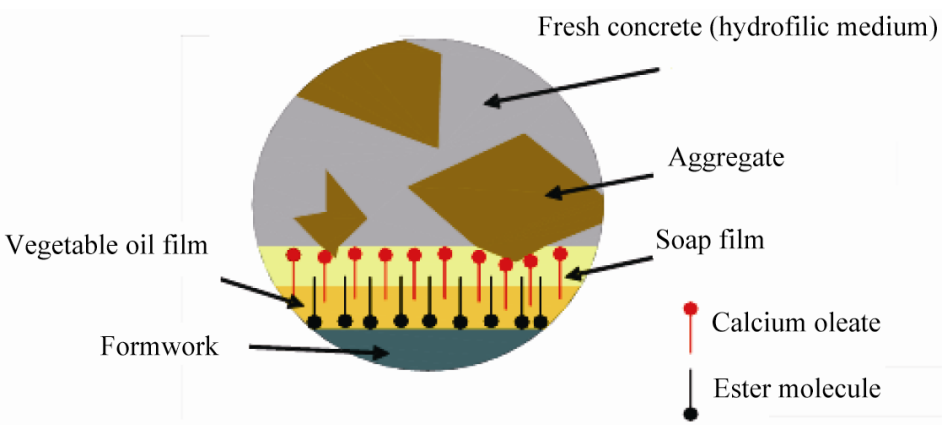

Figure 9. Organisation of a soap film at the concrete/oil/formwork interface. 


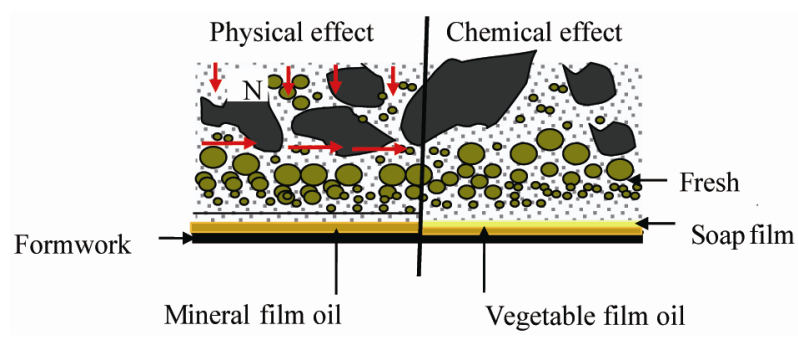

Figure 10. Representation of effects present at the concrete/ oil/formwork interface.

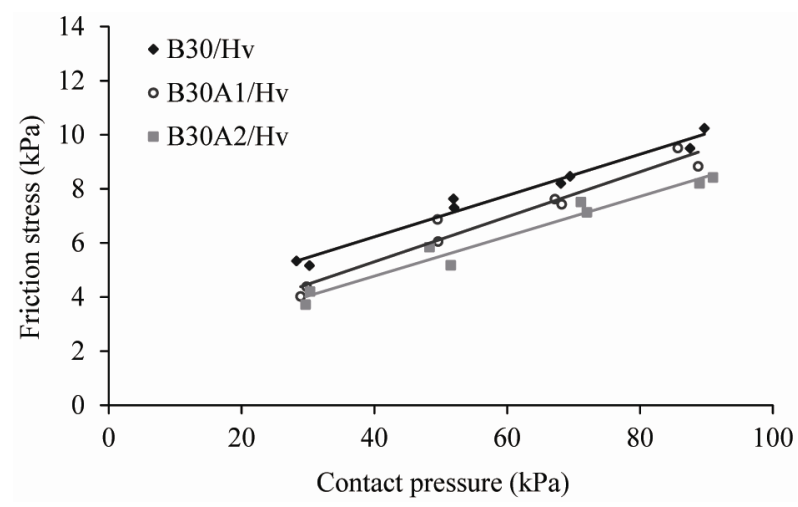

Figure 11. Friction stress as a function of contact pressure for the vegetable release agent.

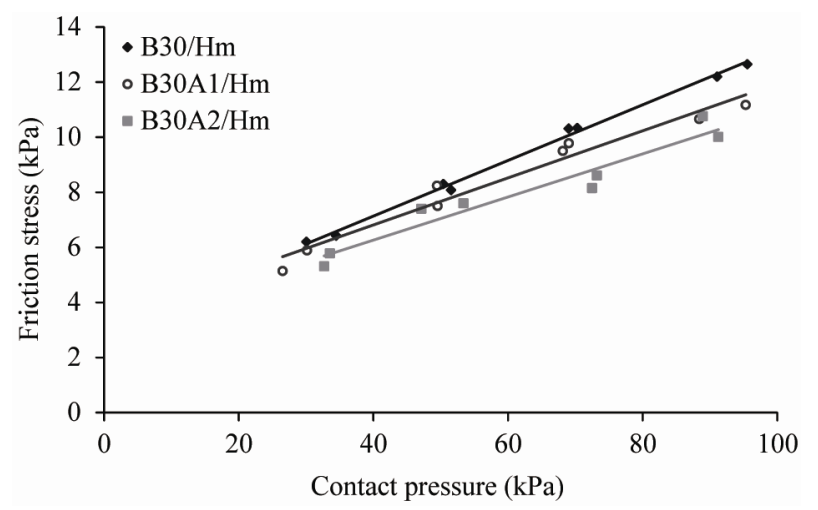

Figure 12. Friction stress as a function of contact pressure for the mineral release agent.

boundary layer, depending on the nature of the release agent. To do this, we studied the tribological behaviour of fresh concrete relative to the form face during concrete placement.

The presence of superplasticizer in the concrete reduces the friction stress at the concrete/formwork interface by making the boundary layer more fluid.

After the sliding of fresh concrete on the formwork, the surfactant power of the superplasticizer stabilises the oil-soap emulsion, thus causing the interstitial medium to thicken. This milky emulsion promotes concrete slide on the oil film, and preserves the "barrier" effect.

Vegetable-based oil retains an advantage over mineral oil, thanks to the double-layer soap-oil organisation. The friction of the fresh concrete against the formwork is so reduced more substantially.

In conclusion, in the vicinity of the formwork face, the superplasticizer acts both on the concrete grains, by deflocculating them, and on the soap-oil micellae, by preventing them from coalescing. Consequently, the performances of superplasticizer depend on the quantity of fines and on the quantity of soap formed. This is why, in order to optimise lubrication performance, the superplasticizer addition rate must be dictated by these two parameters.

\section{REFERENCES}

[1] C. Comparet, "Etude des Interactions Entre les Phases Modèles Représentatives d'un Ciment Portland et des Superplastifiants," Thèse de Doctorat, Université de Bourgogne, Bourgogne, 2004.

[2] C. Djelal, Y. Vanhove and A. Magnin, "Tribological Behaviour of Self-Compacting Concrete,” Cement and Concrete Research, Vol. 34, No. 5, 2004, pp. 821-828. doi:10.1016/j.cemconres.2003.09.013

[3] Y. Vanhove, C. Djelal and A. Magnin, "A Device for Studying Fresh Concrete Friction," Cement Concrete and Aggregates, Vol. 26, No. 2, 2004, pp. 35-41. doi:10.1520/CCA11897

[4] C. Djelal, Y. Vanhove, D. Chambellan and P. Brisset, "Influence of the Application Method of Release Agents on Thickness of Mould Oils," Materials \& Structures, Vol. 43, No. 5, 2010, pp. 687-698. doi:10.1617/s11527-009-9521-z

[5] S. Bethmont, "Mécanismes de Segregation dans les Bétons Autoplaçants (BAP)—Etude Expérimentale des Interactions Granulaires,” Thèse de Doctorat, Ecole Nationale des Ponts et Chaussées, Paris, 2008.

[6] C. Djelal, P. De Caro, L. Libessart and I. Dubois, "Comprehension of Demoulding Mechanics at the Formwork/ Oil/Concrete Interface,” Materials \& Structures, Vol. 41, No. 3, 2008, pp. 571-581. doi:10.1617/s11527-007-9268-3

[7] P. De Caro, C. Djelal, L. Libessart, I. Dubois and N. Pebert, "Influence of the Nature of Demoulding Agent on the Properties Formwork/Concrete/Interface,” Magazine of Concrete Research, Vol. 59, No. 2, 2007, pp. 141-149. doi:10.1680/macr.2007.59.2.141 